\title{
Biophysical stimuli induced by passive movements compensate for lack of skeletal muscle during embryonic skeletogenesis
}

\author{
Niamh C. Nowlan • Gerard Dumas • \\ Shahragim Tajbakhsh • Patrick J. Prendergast • \\ Paula Murphy
}

Received: 5 November 2010 / Accepted: 19 March 2011 / Published online: 20 April 2011

(C) The Author(s) 2011. This article is published with open access at Springerlink.com

\begin{abstract}
In genetically modified mice with abnormal skeletal muscle development, bones and joints are differentially affected by the lack of skeletal muscle. We hypothesise that unequal levels of biophysical stimuli in the developing humerus and femur can explain the differential effects on these rudiments when muscle is absent. We find that the expression patterns of four mechanosensitive genes important for endochondral ossification are differentially affected in muscleless limb mutants, with more extreme changes in the expression in the humerus than in the femur. Using finite element analysis, we show that the biophysical stimuli induced by muscle forces are similar in the humerus and femur, implying that the removal of muscle contractile forces should, in theory, affect the rudiments equally. However, simulations in which a displacement was applied to the end of the limb, such as could be caused in muscleless mice by movements of the mother or normal littermates, predicted higher biophysical stimuli in the femur than in the humerus. Stimuli induced by limb movement were much higher than those induced by the direct application of muscle forces, and we propose that
\end{abstract}

N. C. Nowlan · P. J. Prendergast · P. Murphy

Trinity Centre for Bioengineering, School of Engineering,

Trinity College Dublin, Dublin, Ireland

N. C. Nowlan ( $\varangle)$

EMBL/CRG Systems Biology Unit,

Centre for Genomic Regulation (CRG) and UPF,

Dr. Aiguader, 88, 08003 Barcelona, Spain

e-mail: nowlann@gmail.com

G. Dumas · S. Tajbakhsh

Stem Cells and Development, Department of Developmental

Biology, Institut Pasteur, Paris, France

P. Murphy

Department of Zoology, School of Natural Sciences,

Trinity College Dublin, Dublin, Ireland movements of limbs caused by muscle contractions, rather than the direct application of muscle forces, provide the main mechanical stimuli for normal skeletal development. In muscleless mice, passive movement induces unequal biophysical stimuli in the humerus and femur, providing an explanation for the differential effects seen in these mice. The significance of these results is that forces originating external to the embryo may contribute to the initiation and progression of skeletal development when muscle development is abnormal.

Keywords Skeletal development - Mechanobiology · Mouse mutant · Muscle contractions .

Finite element analysis

\section{Introduction}

Movement during embryogenesis is essential for normal skeletal development. Foetal akinesia deformation sequence (Hall 1986; Hammond and Donnenfeld 1995) results from reduced intrauterine movement, and can lead to craniofacial and limb deformities and abnormal joint contractures. Decreased foetal movement has also been implicated in temporary brittle bone disease in infants, which can lead to multiple unexplained fractures (Miller and Hangartner 1999). Neuromuscular disorders such as congenital myotonic dystrophy (Wesstrom et al. 1986) and spinal muscle atrophy (Nicole et al. 2002), can lead to smaller, thinner and weaker long bones, prone to postnatal fracture (Rodriguez et al. 1988a,b). The relationship between skeletal muscle and development of cartilage, bone and joints is complex; it has been shown that in genetically modified mice with absent or reduced muscle, only some skeletal elements are affected, while other rudiments show no significant 
difference compared to controls (Nowlan et al. 2010a; Gomez et al. 2007; Rot-Nikcevic et al. 2006). Bone development has been examined in two mouse mutants, $M y f 5^{\text {nlacZ/nlacZ }}$ : Myod $^{-/-}$and Pax $3^{S p / S p}$ (Splotch), both of which lack any skeletal muscles. Bones are differentially affected in both of these 'muscleless limb' mutants, with significantly reduced bone formation in the scapula, humerus, ulna and femur but not in the tibia (Nowlan et al. 2010a). The scapula and humerus are the most severely affected with abnormal morphologies of the ossified regions, while ossification centre morphologies develop normally in the ulna, femur and tibia (Nowlan et al. 2008a). In littermates of the double knockouts with one functional copy of Myf5, Myf $5^{\text {nlacZ/+ }}$ : Myod $^{-/-}$, (Rudnicki et al. 1993), muscle mass is reduced by $35-55 \%$. These mice were found to have significantly less bone in the humerus and scapula but no significant difference in bone development in the mutant ulna, femur and tibia (Nowlan et al. 2010a). Joint development can also proceed as normal in the absence of skeletal muscle, but only in some joints (Nowlan et al. 2010a; Kahn et al. 2009). In muscleless mutants, the elbow fails to undergo cavitation (Nowlan et al. 2010a; Kahn et al. 2009), the shoulder undergoes partial cavitation (Nowlan et al. 2010a), while normal joint development is observed in the knee (Nowlan et al. 2010a; Kahn et al. 2009) and digit joints (Kahn et al. 2009), despite the lack of skeletal muscle. In contrast to results from muscleless limb mice, there have been no differential effects reported for joint development in immobilised chicks (Nowlan et al. 2010b), as all synovial joints examined have been found to be affected by the lack of muscle contractions (Drachman and Sokóloff 1966; Ruano-Gil et al. 1978; Osborne et al. 2002; Mitrovic 1982). Furthermore, while the skeletal rudiments of immobilised chicks have been found to be affected to varying degrees of severity (Hall and Herring 1990), no rudiment in the chick has been reported as being unaffected by immobilisation (Hall and Herring 1990; Murray and Drachman 1969; Hosseini and Hogg 1991).

While movement due to spontaneous muscle contractions before birth (or hatching) is common to all vertebrate embryos, the mechanical environment of the developing embryo will also be affected by external factors. While the mouse embryo may be subjected to frequent external mechanical stimulation due to maternal and littermate movements, the chick embryo will experience only the forces that result from the egg being periodically turned. Passive movements due to external forces have previously been identified as a potential source of imposed stresses in the early embryo (Henderson and Carter 2002), but their influence on morphogenesis has not been considered in detail. In muscleless limb mice, it is possible that movements of the mother or (normal) littermates could induce biophysical stimuli, despite the lack of spontaneous muscle contractions within the mutant embryo. While the effects of active or passive movements on the mechanical environment in utero cannot easily be measured directly, finite element analysis can be used to predict patterns and levels of biophysical stimuli in the developing embryo. Finite element analysis has previously been used to investigate the influence of muscle forces on skeletal development in studies on ossification (Nowlan et al. 2008a,b; Tanck et al. 2000; Carter et al. 1987; Stevens et al. 1999), sesamoid formation (Sarin and Carter 2000), and joint development (Shefelbine and Carter 2004; Heegaard et al. 1999; Roddy et al. 2011a,b). Finite element analyses of bone collar development in the chick predicted co-localisation of peak biophysical stimuli levels induced by muscle forces with presumptive regions of periosteal bone formation (Nowlan et al. 2008a), and also with the expression patterns of two genes involved in bone development; Collagen10a1 (ColX) and Indian Hedgehog (Ihh) (Nowlan et al. 2008b). When developing chicks were immobilised, the predicted decreases in biophysical stimuli levels correlated with the decreased bone formation and the changes in the expression patterns of ColX and Ihh found experimentally (Nowlan et al. 2008b), demonstrating the predictive power of such simulations.

In this study, we investigate the mechanism underlying the differential effects on the developing murine skeleton when limb muscle is reduced or absent. The humerus is more affected than the femur (Nowlan et al. 2010a), and we focus our study on a comparison of these two rudiments. We hypothesise that unequal levels of biophysical stimuli can explain the differential effects of absent musculature on these rudiments. We first characterise the expression patterns of five mechanosensitive genes that are known to regulate skeletal development (Provot and Schipani 2005) in muscleless limb mice, in order to investigate whether mechanosensitive gene expression patterns can be used to detect differences in the local mechanical environments of rudiments in the developing skeleton. Based on previous evidence of the in vivo mechanosensitivity of ColX and Ihh (Nowlan et al. 2008b), we compare these genes between the humeri and femora of muscleless limb mouse mutants, and we extend our examination to include fibroblast growth factor receptor 3 (FGFr3) (Shiang et al. 1994), parathyroid hormone-related protein (PTHrP) (Lanske et al. 1996) and runt-related transcription factor 2 (Runx2) (Yoshida et al. 2002), all of which have been shown to be mechanosensitive in vitro (Jackson et al. 2006; Ng et al. 2006; Goldring et al. 2006; Tanaka et al. 2005; Sundaramurthy and Mao 2006). More extreme changes in the expression pattern of one or more mechanosensitive genes in the humerus than in the femur would indicate a potential difference between the underlying mechanical environments of the two rudiments. We then use finite element analysis to predict the biophysical stimuli induced by muscle contraction forces (in normal mice), and those induced by passive movements of the limbs due to maternal and littermate 
movements, and compare these stimuli levels between the humerus and femur. If the differential effects on the humerus and femur are detectable in the expression patterns of mechanosensitive genes, and in the levels of biophysical stimuli induced by muscle contractions and/or passive movement, this would indicate that the differential effects on skeletogenesis in muscleless mice may be due to mechanobiological factors.

\section{Methods}

\subsection{Gene expression analysis}

Myf $5^{\text {nlacZ } /+}:$ Myod $^{+/-}$or Pax $3^{\text {Sp } /+}$ mice were interbred, and offspring were subsequently genotyped as described previously (Kassar-Duchossoy et al. 2004; Tajbakhsh et al. 1997). Embryos and foetuses were harvested between E13.5 and E15.5, and each embryo was staged using Theiler morphological criteria (Theiler 1989). Spontaneous limb movements have been shown to occur in ex utero E12.5 embryos (Suzue 1996). A total of 25 pairs of muscleless limb mutants and stage-matched controls were obtained; 16 of which were at stage TS23. These were divided into five groups, and each group was assigned to one gene, meaning at least three mutant and three control TS23 embryos were analysed for each of 5 genes. Two pairs of mutants and control embryos at TS22, three pairs at TS24 and four pairs at TS25 were also obtained and divided for analysis. The right forelimb and hindlimb of each animal were sectioned longitudinally using a vibrating microtome and analysed for the expression of the assigned candidate gene by in situ hybridisation as described previously (Nowlan et al. 2008b). The five genes characterised in control and mutant animals were ColX, Ihh, FGFr3, PTHrP and Runx2. The probe generated for ColX was obtained from IMAGE ${ }^{1}$ clone \#30758452 and aligns with nucleotides 915-2729 on Genbank sequence ref NM_009925.3. The probe generated for FGFr3 was produced from IMAGE clone \#5708838 and aligns with nucleotides 89-4154 on Genbank sequence ref NM_008010. The probe generated for Ihh was produced from a clone obtained from Prof. R. E. Hill (MRC Human Genetics Unit, Edinburgh) and aligns with nucleotides 1-3127 on Genbank sequence ref NM_010544.2. The probe generated for PTHrP was produced from IMAGE clone \#5346064 and aligns with nucleotides 287-1583 on Genbank sequence ref NM_008970.1. The probe generated for Runx 2 was produced from IMAGE clone F730001N14 and aligns with nucleotides 262-3442 on Genbank sequence ref NM_009820.2.

\footnotetext{
1 http://www.image.hudsonalpha.org/, last accessed February 2011.
}

\subsection{Finite element analyses}

\subsubsection{Limb and rudiment mesh construction}

Two types of finite element analyses were performed; the first simulating the effect of muscle contraction forces on the elements of the proximal forelimb and hindlimb (i.e. the humerus and femur), and the second modelling the effect of a distal displacement on the whole limb, representing passive movement of the limb. The finite element analyses were performed in Abaqus. ${ }^{2}$ The meshes used for the two simulation types, although constructed in different ways, were obtained from the same specimens as follows. Control mouse embryos were harvested and staged according to the Theiler Staging system (Theiler 1989). Embryos corresponding to typical TS22, TS23 and TS24 stages were selected for analysis and were stained for cartilage and bone using Alcian Blue and Alizarin Red as described by Nowlan et al. (2010a). Stained limbs were scanned using optical projection tomography (OPT, Sharpe et al. 2002) to give 3-D representations of the cartilage and bone in each skeletal element. At least four forelimbs and hindlimbs per stage were stained and scanned, and the left limbs of two animals per stage were selected for finite element analysis. To confirm the precision of the 3-D scans of bone and cartilage, stained limbs (at least a further four per stage) were sectioned using a vibratome (VT1000S, Leica) and photographed. In addition, unstained limbs were sectioned and then stained to verify the efficacy of the wholemount staining method.

The humerus and femur were modelled at TS22, TS23 and TS24. The 'whole limb' analyses were performed at TS22 and TS23 only, due to the likelihood of significant joint articulation at TS24. Morphologies obtained from two different animals at each stage were modelled, giving a total of six models of the humerus and six of the femur, four models of the forelimb and four of the hindlimb. The meshes for finite element analysis of the humerus and femur were obtained from the OPT data using the Rhino ${ }^{3}$ and Cubit ${ }^{4}$ software programs, as described previously (Nowlan et al. 2008a), and the sizes of the meshes used for each stage are detailed in Table 1. The whole limb analyses were performed with simplified meshes of all but the most distal part (the autopod) of each limb, as shown in Fig. 1. The forelimb meshes were constructed from the scapula, humerus, radius and ulna, and the hindlimb meshes from the pubic ramus, femur, tibia and fibula. Pre-processing in Rhino was performed in order to simplify the limbs into one contiguous shape, where the joint regions were represented as continuous regions in the

\footnotetext{
2 C), http://www.simulia.com/, last accessed February 2011.

3 C , http://www.rhino3d.com/, last accessed February 2011.

4 @ , http://www.cubit.sandia.gov/, last accessed February 2011.
} 
Table 1 Lengths of humerus and femur meshes, in $\mathrm{mm}$

\begin{tabular}{llllll}
\hline Stage & Humerus & & & Femur \\
\cline { 2 - 3 } \cline { 6 - 6 } & Animal \#1 & Animal \#2 & & Animal \#1 & Animal \#2 \\
\hline TS22 & 2.13 & 2.18 & & 1.8 & 1.77 \\
TS23 & 2.72 & 2.87 & & 2.18 & 1.9 \\
TS24 & 3.27 & 3.14 & & 3.24 & 3.07 \\
\hline
\end{tabular}

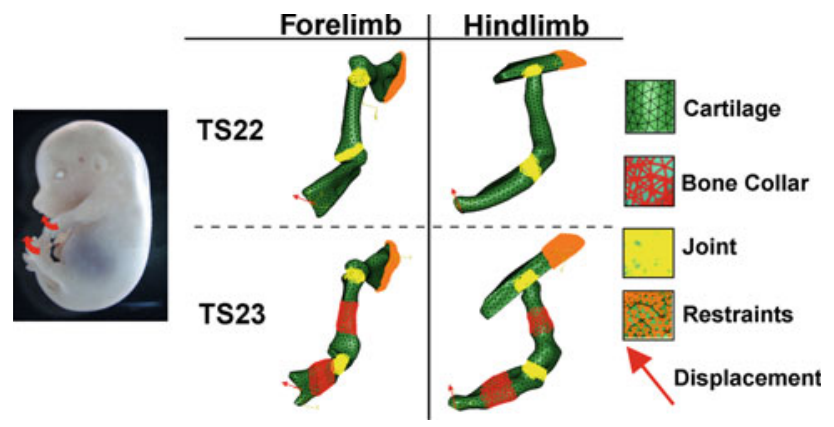

Fig. 1 Lateral view of TS23 embryo (left) with views of forelimb and hindlimb meshes from Animal \#1. Assigned joint regions are highlighted in yellow and bone collar regions in red. The most posterior part of the scapula (forelimb) and the posterior part of the pubic ramus (hindlimb) were restrained from movement in any direction, as shown in orange. Red arrow indicates direction of displacement modelled

limb and the rudiments of the zeugopod were amalgamated into one region, as illustrated in Fig. 1.

\subsubsection{Loads}

In order to calculate the muscle forces acting on the developing humerus and femur, developing muscles were visualised at TS22, TS23 and TS24 through X-Gal staining of embryos carrying the $\beta$-galactosidase gene insertion in a single Myf5 locus, as described by Tajbakhsh et al. (1996). X-Gal staining was performed as described previously (Tajbakhsh et al. 1996) on a separate set of embryos from those stained for cartilage and bone, and 3-D images of the muscles were obtained by scanning X-Gal-stained embryos with a $700 \mathrm{~nm}$ visible light filter using OPT. At least four forelimbs and hindlimbs were scanned at each stage, and for each of the stages of interest, the two animals best corresponding to the Theiler Stage criteria were used for muscle area measurement. The Mouse Limb Atlas (DeLaurier et al. 2008), an interactive 3 -D model of the bones, muscles and tendons in the TS23 mouse forelimb and hindlimb, also generated from OPT data, was used to determine which muscles act on each part of the limb, and the line of action and point of application of each muscle force. After verifying that the same muscle bodies are present at TS22 and TS24 as at TS23, it was assumed that the muscle attachment sites shown in the Mouse Limb Atlas at TS23 are consistent with those at TS22 and TS24.
The cross-sectional areas of easily identifiable muscle bodies were measured perpendicular to the muscle working direction from the 3-D OPT muscle data, with results averaged from two specimens at each stage. By relating muscle measurements from the OPT data and the Mouse Limb Atlas (DeLaurier et al. 2008), cross-sectional areas (perpendicular to the working direction, in $\mathrm{mm}^{2}$ ) for all muscles were determined. As described previously, (Nowlan et al. 2008a), a force per unit area value for embryonic muscle (Landmesser and Morris 1975) was used to estimate the force exerted by each individual muscle. The muscles active at each stage and location, with the measured areas and calculated forces, are detailed in Table 2. Two sequential muscle contractions lasting $1.4 \mathrm{~s}$ each were modelled, an extension contraction of the muscles on the posterior aspect of each rudiment followed by a flexion of the muscles on the anterior aspect of each rudiment, as performed previously (Nowlan et al. 2008a; Tanck et al. 2000). In order to verify that the results were not dependent on this sequential activation of the muscles, models were also run in which all muscles were activated simultaneously. Muscle forces were applied at the distal ends of the rudiments in the form of a surface traction over one or more element surfaces, depending on the size of the insertion site as estimated from the Mouse Limb Atlas (DeLaurier et al. 2008). Muscle force applications sites on the humerus and femur are shown for the three stages examined in Fig. 2.

For the whole limb analyses, a displacement of $10 \mu \mathrm{m}$ was applied to the distal end of the zeugopod, towards the body of the embryo, as shown in Fig. 1. This displacement was assumed to result from external forces applied directly through the uterine wall and amnion, due to movements of the mother or littermates, with the most likely direction of the applied displacement based on the position of the limbs at this stage, as shown in Fig. 1. The displacement was applied for $1.4 \mathrm{~s}$ with ramp-up, hold and ramp-down phases. Additional analyses were also performed in which the displacement was imposed in alternative directions; in the anterior to posterior and the posterior to anterior directions with respect to the main body (head to tail) axis. Displacements of 1 and $50 \mu \mathrm{m}$ were also applied (in the standard dorsal-ventral direction) for comparison purposes. Further analyses were carried out where a force of $5 \mathrm{mN}$, instead of a displacement, was applied to the distal end of the zeugopod.

\subsubsection{Material properties}

The Young's moduli of murine embryonic cartilage and mineralised tissue as measured by Tanck et al. (2004) were used, with detailed mechanical properties as defined in Table 3. At TS22, the humerus and femur are composed of cartilage only. At TS23, both rudiments have periosteal bone at the middiaphysis, which was represented by a bone collar to a depth of $0.1 \mathrm{~mm}$ (Nowlan et al. 2008a), where the length of the bone 
Table 2 Muscles applied to humerus in forelimb (FL) and femur in hindlimb (HL) with measured area, and calculated force values used

\begin{tabular}{|c|c|c|c|c|c|c|c|c|}
\hline Rudiment & Name & Step & $\begin{array}{l}\text { Area TS22 } \\
(\mathrm{sq} \mathrm{cm})\end{array}$ & $\begin{array}{l}\text { Force TS22 } \\
(\mu N)\end{array}$ & $\begin{array}{l}\text { Area TS23 } \\
(\mathrm{sq} \mathrm{cm})\end{array}$ & $\begin{array}{l}\text { Force TS23 } \\
(\mu \mathrm{N})\end{array}$ & $\begin{array}{l}\text { Area TS24 } \\
(\mathrm{sq} \mathrm{cm})\end{array}$ & $\begin{array}{l}\text { Force TS24 } \\
(\mu \mathrm{N})\end{array}$ \\
\hline Humerus & Triceps brachii (lateral) & Extension & 383 & 42.5 & 587 & 65.1 & 1116 & 123.9 \\
\hline Humerus & Triceps brachii (long) & Extension & 929 & 103.1 & 1424 & 158.1 & 2709 & 300.7 \\
\hline \multirow[t]{2}{*}{ Humerus } & Triceps brachii (medial) & Extension & 170 & 18.9 & 261 & 29.0 & 497 & 55.2 \\
\hline & Total (humerus, extension) & & & 164.5 & & 252.2 & & 479.8 \\
\hline Humerus & Acromiodeltoidus & Flexion & 53 & 5.8 & 81 & 8.9 & 153 & 17.0 \\
\hline Humerus & Biceps brachii (long) & Flexion & 158 & 17.5 & 242 & 26.9 & 461 & 51.1 \\
\hline Humerus & Biceps brachii (short) & Flexion & 46 & 5.2 & 71 & 7.9 & 135 & 15.0 \\
\hline \multirow[t]{2}{*}{ Humerus } & Brachialis & Flexion & 216 & 24.0 & 331 & 36.7 & 630 & 69.9 \\
\hline & Total (humerus, flexion) & & & 46.7 & & 71.5 & & 136 \\
\hline Femur & Vastus intermedius & Extension & 470 & 52.2 & 808 & 89.7 & 1506 & 167.2 \\
\hline Femur & Vastus lateralis & Extension & 533 & 59.2 & 918 & 101.9 & 1709 & 189.8 \\
\hline \multirow[t]{2}{*}{ Femur } & Vastus medialis & Extension & 265 & 29.5 & 457 & 50.7 & 851 & 94.4 \\
\hline & Total (femur, extension) & & & 140.9 & & 242.3 & & 451.4 \\
\hline Femur & Adductor brevis & Flexion & 175 & 19.5 & 302 & 33.5 & 562 & 62.4 \\
\hline Femur & Adductor magnus & Flexion & 123 & 13.6 & 211 & 23.5 & 394 & 43.7 \\
\hline Femur & Biceps Femoris & Flexion & 366 & 40.6 & 630 & 69.9 & 1173 & 130.2 \\
\hline Femur & Pectineus + adductor longus & Flexion & 306 & 34.0 & 527 & 58.5 & 981 & 108.9 \\
\hline \multirow[t]{2}{*}{ Femur } & Semimembranosus & Flexion & 366 & 40.6 & 630 & 69.9 & 1173 & 130.2 \\
\hline & Total (femur, flexion) & & & 115.2 & & 198.3 & & 369.3 \\
\hline
\end{tabular}

Total muscle forces for each contraction are given in bold

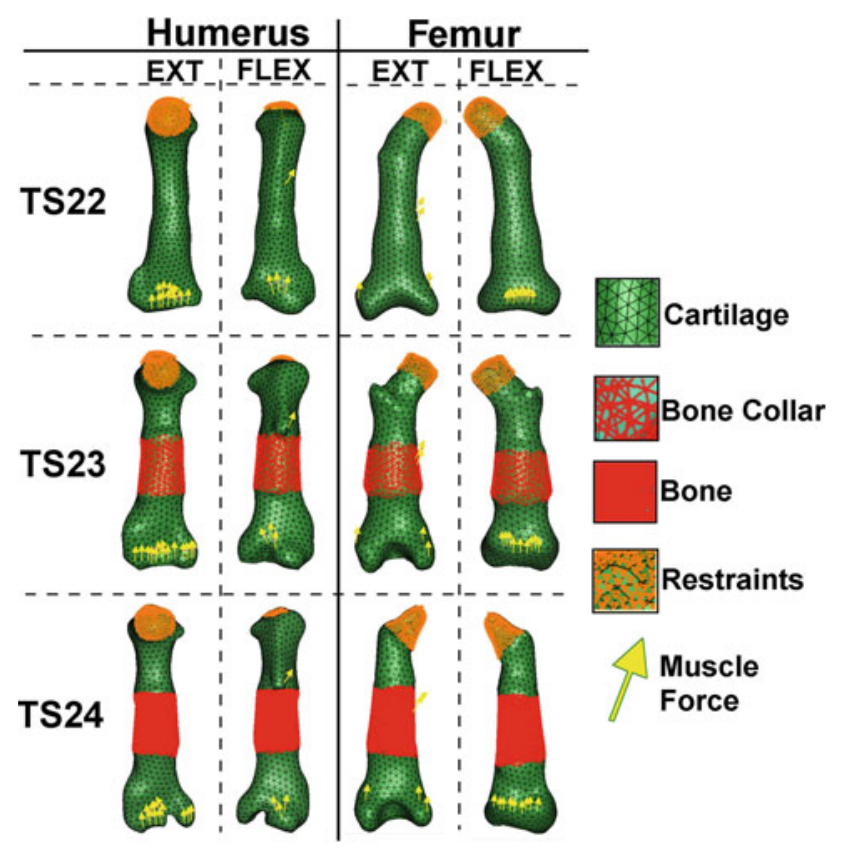

Fig. 2 Material property assignments, boundary conditions and applied muscle loads in humerus and femur models. Muscle forces on the posterior aspect were activated during the extension (EXT) contraction, and muscle forces on the anterior aspect were activated during the flexion (FLEX) contraction collar was evident from the imported cartilage and bone OPT data. At TS24, both rudiments contain a central solid mineralised region, the extent of which was again determined from the imported cartilage and bone data. Material properties for the humerus and femur models are illustrated in Fig. 2. Bone collars were also included on the humerus and femur in the whole limb analyses, and also on the zeugopod regions, as illustrated in Fig. 1. In the whole limb analyses, joint regions were assigned a Young's modulus of half that of the cartilage $(0.55 \mathrm{MPa})$ to represent the interzone region, based on data from chick interzone tissue (Roddy et al. 2011a). The regions assigned joint material properties are illustrated in Fig. 1, and material properties are detailed in Table 3. As we are unaware of any published data on the mechanical properties of the mammalian interzone region, we also ran the models with cartilage material properties in the interzones in order to assess the impact of the joint region material properties on the model results.

\subsubsection{Boundary conditions}

A zero pore-pressure boundary condition was specified on the external nodes of all meshes, thereby allowing fluid flow out of the structure. The humerus and femur models were modelled as if no movement occurred at the proximal end of the rudiment, implemented by fixing the surface nodes at the proximal femur and humerus in all directions. In the humerus, 
Table 3 Material properties with source data from (1) Tanck et al. 2004 and (2) Lacroix and Prendergast (2002), as marked

\begin{tabular}{llll}
\hline & Cartilage & Bone & Joint \\
\hline Young's modulus (MPa) & $1.1(1)$ & $117(1)$ & 0.55 \\
Permeability $\left(\mathrm{m}^{4} / \mathrm{Ns}\right)$ & $6.7 \mathrm{E}-15(1)$ & $6.7 \mathrm{E}-16(1)$ & $6.7 \mathrm{E}-15(1)$ \\
Poisson's ratio & $0.25(1)$ & $0.3(1)$ & $0.25(1)$ \\
Solid compression modulus (MPa) & $2300(2)$ & $13920(2)$ & $2300(2)$ \\
Fluid compression modulus (MPa) & $2300(2)$ & $2300(2)$ & $2300(2)$ \\
Porosity & $0.8(2)$ & $0.8(2)$ & $0.8(2)$ \\
\hline
\end{tabular}

surface nodes located ventral to the developing lesser tuberosity were fixed, while the nodes proximal to the greater trochanter were fixed in the femur, as illustrated in Fig. 2. For the whole limb analyses, in the forelimb, the most posterior region of the scapula was restrained completely, while in the hindlimb, the posterior part of the pubic ramus was restrained from movement in any direction (Fig. 1). For each analysis, octahedral strain and relative fluid-solid velocity were computed. A stimulus $(S)$ was also defined, following Prendergast et al. (1997), as a combination of the shear strain and fluid velocity:

$S=\frac{\gamma_{o c t}}{a}+\frac{v}{b}$

where $a=0.0375$, and $\mathrm{b}=3 \mu \mathrm{ms}^{-1}$.

\section{Results}

3.1 Changes in mechanosensitive gene expression patterns in muscleless limbs

While abnormal expression patterns were seen for all five genes in mutant limbs, four out of five genes, ColX, FGFr3,
Ihh and Runx2, showed more pronounced changes in the expression pattern in the humerus than in the femur (Fig. 3), while PTHrP appeared to have the same changes in the expression in both the forelimb and hindlimb (Fig. 4). At TS23, ColX expression in the control humerus and femur has split into two regions proximal and distal to the mid-diaphysis, (Fig. 3a). However, all mutant (one $P a x 3^{S p / S p}$ and two Myf $5^{\text {nlacZ/nlacZ }}:$ Myod $^{-/-}$) forelimbs at TS23 showed a decrease in the domain of ColX and some showed no split in ColX expression (Fig. 3a), while in a $P a x 3^{S p / S p}$ mutant femur, the decreases in the expression domains were not as pronounced as in the humeri (Fig. 3b). By TS24, two regions of ColX expression were present in a TS24 $P a x 3^{S p / S p}$ humerus and femur and a TS25 $P a \times 3^{S p / S p}$ humerus, but with a decreased distance between regions of expression as compared to the controls, the decrease being more pronounced in the humerus than in the femur at TS24 (humeral data shown in Fig. 5a-d). ColX expression in mutant humeri at TS24 and TS25 resembled the expression patterns at earlier stages in control rudiments (compare Figs. 5b to 3a), indicating that ColX expression in mutant limbs appears to be lagging behind that of control limbs, with a more pronounced delay in the humerus than in the femur. At TS23, FGFr3 is normally expressed throughout the humerus

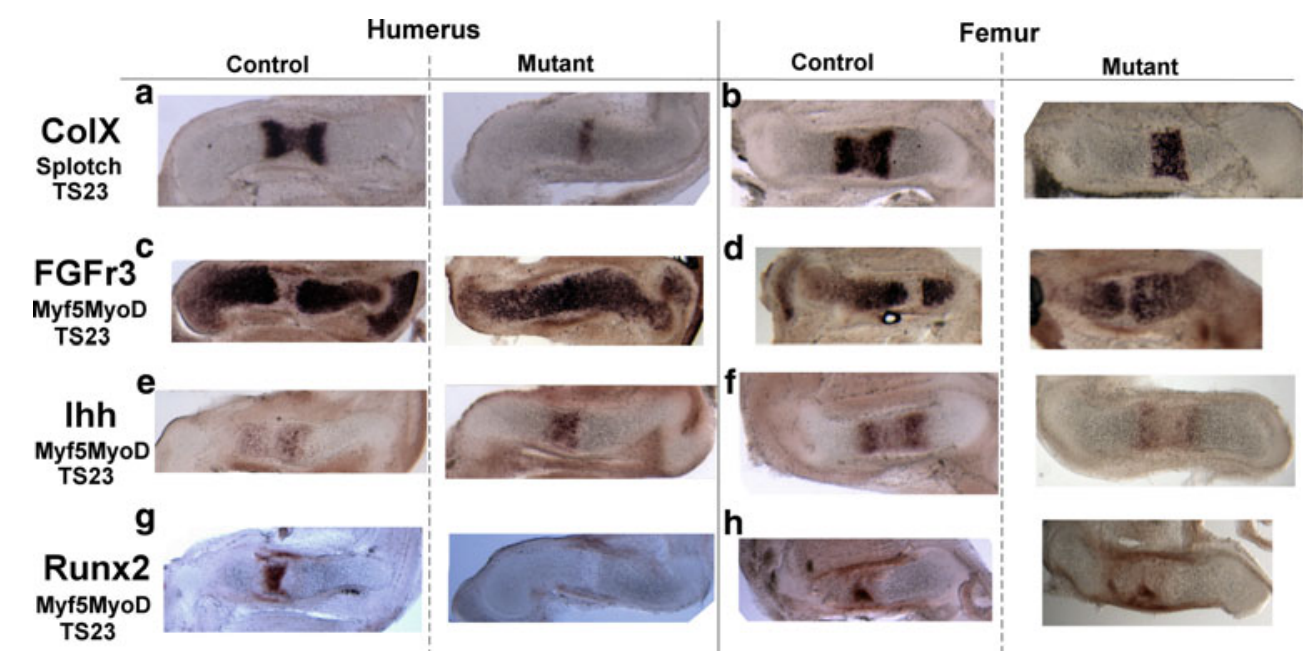

Fig. 3 Expression patterns of ColX, FGFr3, Ihh and Runx2 in control and muscleless $\left(M y f 5^{\text {nlacZ/nlacZ }}: M_{y o d}{ }^{-/-}:\right.$Myf5MyoD, Pax3 $3^{S p / S p}$ : Splotch) humeri and femora at TS23. Sections oriented with proximal end to left, and anterior aspect up 


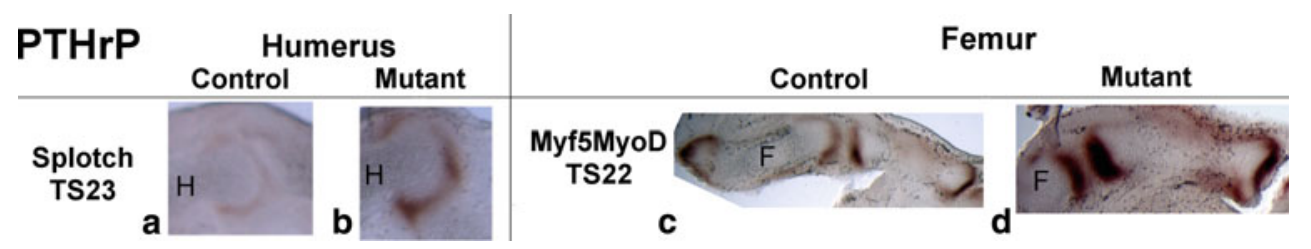

Fig. 4 PTHrP expression patterns in elbow (humerus indicated by "H") in TS23 control (a) and $P a x 3^{S p / S p}$ : Splotch (b) and in hindlimbs of TS22 Myf $5^{\text {nlacZ/nlacZ }}:$ Myod $^{-/-}(\mathrm{d})$ and littermate control

(c, distal end of femur shown for mutant, femur indicated by "F"). Sections oriented with proximal end to left, and anterior aspect up

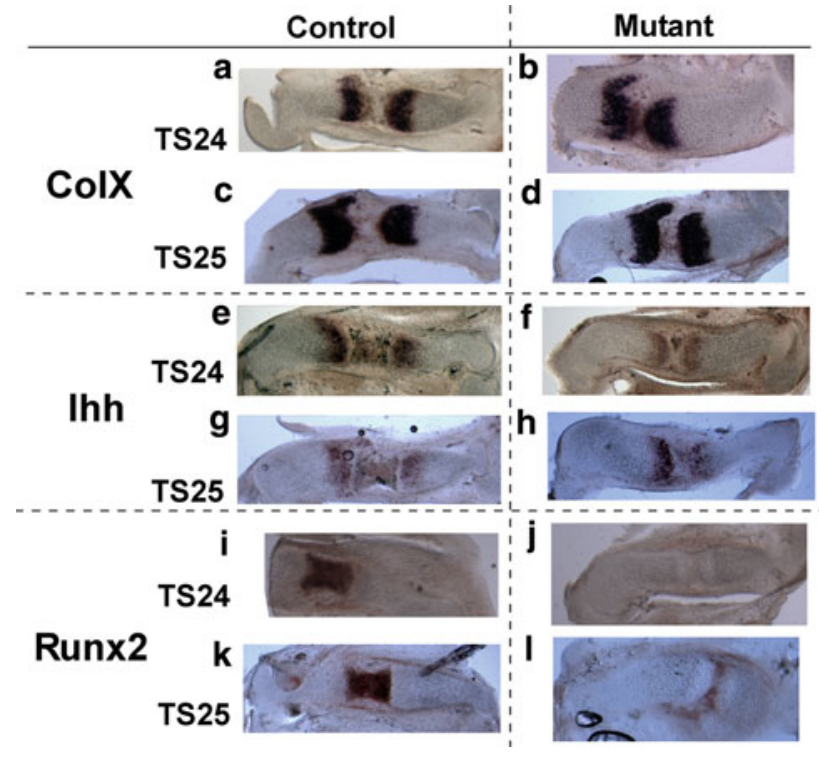

Fig. 5 Expression patterns of ColX, Ihh and Runx2 in control and muscleless mutant (all $P a \times 3^{S p / S p}$ : Splotch) humeri at TS24 and TS25. Sections oriented with proximal end to left, and anterior aspect up

and femur except for a gap at the mid-diaphysis (Fig. 3c, d). Either no break in the expression or a much reduced gap at the mid-diaphysis was seen in three mutant (one $P a \times 3^{S p / S p}$ and two $M y f 5^{\text {nlac }} /$ nlacZ $: M$ yod $^{-/-}$) humeri (Fig. 3c), while both mutant (one $P a x 3^{S p / S p}$ and one $M y f 5^{\text {nlacZ/nlacZ }}$ : Myod $^{-/-}$) femora displayed gaps in FGFr3 expression at the mid-diaphysis (Fig. 3d) with one of these being indistinguishable from controls (data not shown). While control humeri at TS22 and TS23 have two regions of Ihh expression proximal and distal to the mid-diaphysis in the forming growth plates, only one mid-diaphyseal region of Ihh expression was detectable in all TS22 and TS23 Myf $5^{\text {nlacZ } / \text { nlac Z }}$ : Myod $^{-1-}$ humeri (TS23, Fig. 3e). Ihh expression patterns in $M y f 5^{\text {nlacZ/nlacZ }}:$ Myod $^{-/-}$femora at TS22 and TS23 were not as severely affected, with normal separation of expression regions (TS23, Fig. 3f). $P a \times 3^{S p / S p}$ humeri at TS24 and TS25 did display two regions of Ihh expression, but with greatly reduced separation between the growth plate expression domains as compared to the stage-matched controls (Fig. 5e-h), and expression patterns in mutant humeri did not match those of control limbs at earlier stages (compare Figs. $5 \mathrm{f}$ to $3 \mathrm{e}$ and Fig. $5 \mathrm{~h}$ to e), indicating that the effect on Ihh expression is more complex than a delay. The normal pattern for Runx 2 at TS23 is a region of expression in the cartilage at the mid-diaphysis and in the perichondrium adjacent to, and extending beyond, the mid-diaphyseal region of expression (Fig. 3g, h). Three $M y f 5^{\text {nlacZ/nlacZ }}:$ Myod $^{-/-}$ humeri at TS23 showed altered expression, with either a reduced extent of expression at the mid-diaphyseal core or a complete absence of expression in this region (Fig. $3 \mathrm{~g}$ ). Two Myf $5^{\text {nlacZ/nlacZ }}$ : Myod $^{-/-}$femora at TS23 exhibited either no apparent difference to the expression pattern in the control femur (Fig. 3h) or a decreased region of expression at the core as compared to the controls. At TS24 and $\mathrm{TS} 25, P a x 3^{S p / S p}$ humeri also had a greatly decreased region of Runx2 expression at the core in comparison with control stage-matched humeri (Fig. 5i-1), while Runx2 expression in the $P a \times 3^{S p / S p}$ femur at TS24 was similar to that of the stage-matched control (data not shown). As was seen for Ihh, Runx 2 expression patterns in the humerus at later stages did not match earlier control stages (compare Figs. 5j to $3 \mathrm{~g}$ and Figs. 51 to $\mathrm{i}$ ), indicating that the lack of muscle did not simply delay progression of Runx2 expression. PTHrP is normally expressed in periarticular cartilage at the joint interface (Fig. $4 \mathrm{a}, \mathrm{c}$ ). In all $P a x 3^{S p / S p}$ and $M y f 5^{\text {nlacZ/nlacZ }}$ : Myod $^{-1-}$ forelimbs and hindlimbs, the intensity and apparent extent of PTHrP-stained regions were increased relative to control limbs, as shown for a TS23 Pax $3^{S p / S p}$ elbow joint (Fig. 4b) and a TS22 Myf $5^{\text {nlacZ/nlacZ }}:$ Myod $^{-/-}$mutant hindlimb (Fig. 4d), with no apparent difference in severity between mutant femora and humeri.

\subsection{Biophysical stimuli resulting from muscle contractions}

Levels of fluid flow, octahedral shear strain and stimulus (a combined measure of octahedral shear strain and fluid flow) peaked at the mid-diaphysis, or proximal to the middiaphysis at TS22, and proximal and distal to the ossified regions at TS23 and TS24, as shown in Fig. 6. Results shown are for animal \#1 and are from $0.4 \mathrm{~s}$ into the extension contraction, at the start of the hold phase, and are presented as external posterior and anterior views, and as virtual sections 

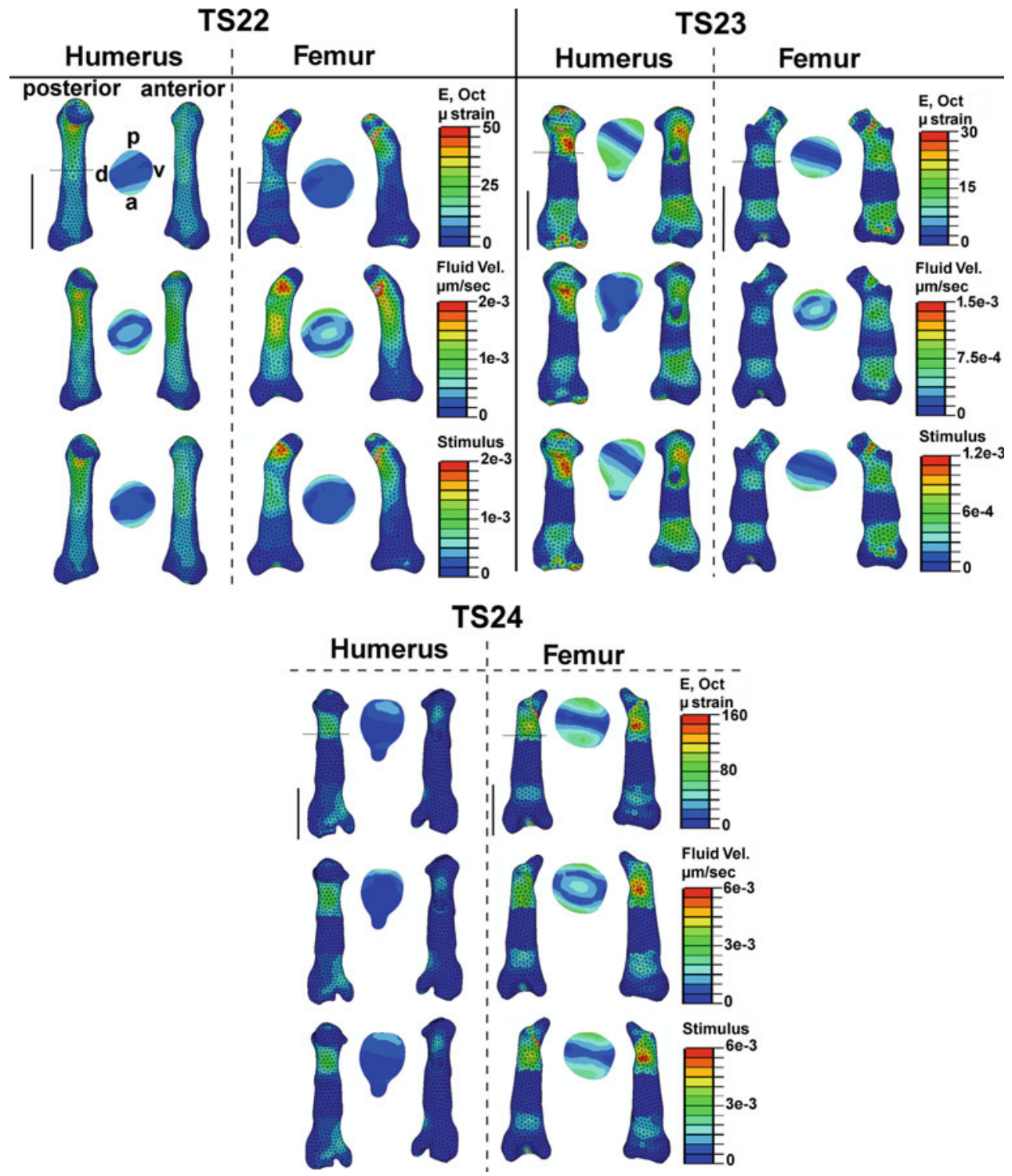

Fig. 6 Comparison of Octahedral strain (E, Oct), Fluid velocity and Stimulus (as described in text) incurred by muscle contractions during the extension contraction $(t=0.4 \mathrm{~s})$ in humeri and femora at stages TS22, TS23 and TS24. Stimuli displayed as external posterior and

through the presumptive bone regions in Fig. 6. Regions of presumptive bone formation are those regions of cartilage that would undergo ossification within the next $24 \mathrm{~h}$ and correspond with the zones of hypertrophic chondrocytes in the growth plates. Virtual sections through these regions were taken at the mid-diaphysis at TS22, proximal to the bone collar at TS23 and proximal to the mineralised region at TS24 as indicated with a dashed line on the external views in Fig. 6. Stimuli magnitudes tended to be higher in the proximal end of the rudiments, which was likely due to the proximal fixation and the application of loads to the distal end (Fig. 2). The predicted magnitudes of biophysical anterior views and through sections at presumptive bone regions (location marked with dotted horizontal line) displayed. Section orientations shown at top left, $a$ anterior, $p$ posterior, $d$ dorsal, $v$ ventral. Vertical scale bars (external views) $1 \mathrm{~mm}$

stimuli induced by muscle contractions were low, with peak octahedral shear strain values ranging from $13-20 \mu$ strain at TS22 and TS23, to $110-140 \mu$ strain at TS24 and fluid velocities ranging from $1.0-1.5 \times 10^{-3} \mu \mathrm{m} / \mathrm{s}$ at TS22 and TS23, to $3.8-4.4 \times 10^{-3} \mu \mathrm{m} / \mathrm{s}$ at TS24 in the presumptive bone regions. It is likely that the higher stimuli magnitudes at TS24 are due to the solid calcified region at the mid-diaphysis at this stage, transferring loads to the adjacent cartilage. During the flexion contraction, patterns of biophysical stimuli were similar while stimuli magnitudes were lower than during the extension contraction (data not shown), due to the lower muscle forces during this phase (Table 2). 


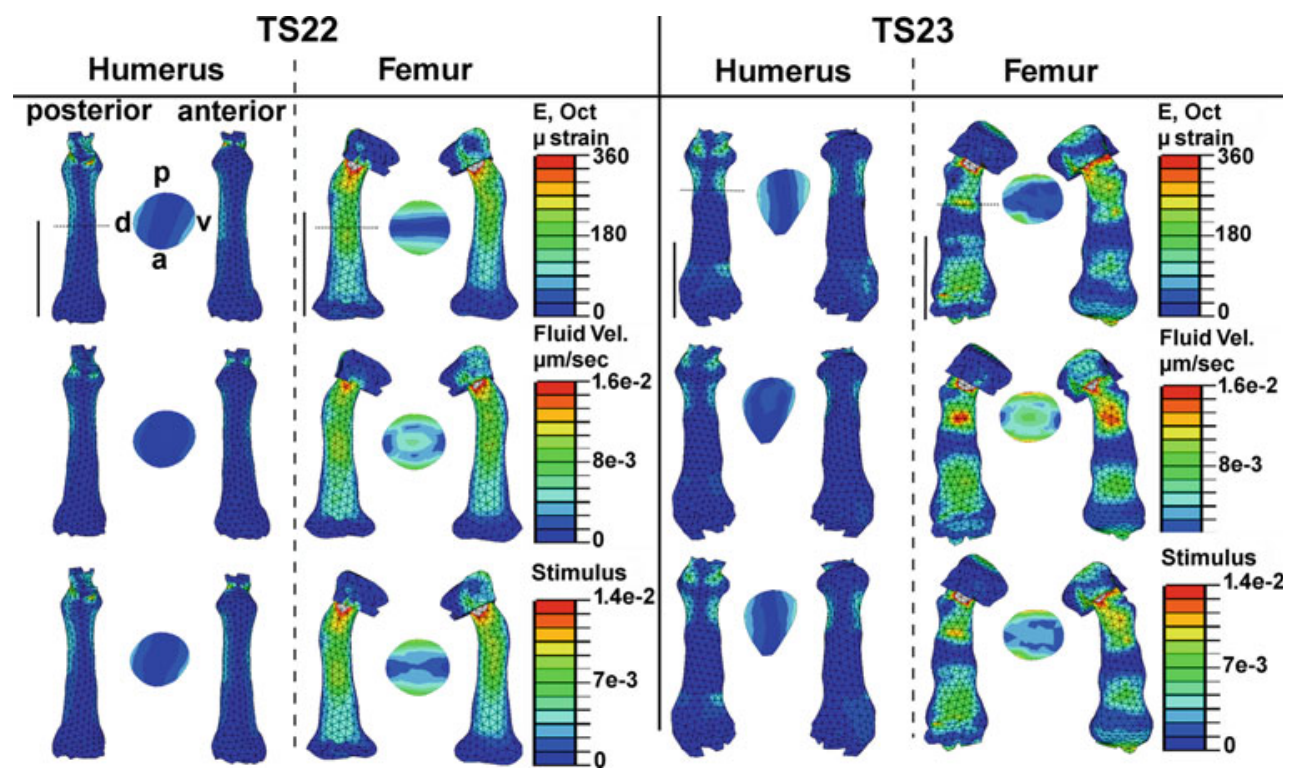

Fig. 7 Comparison of biophysical stimuli incurred in the humerus and femur by a $10 \mu \mathrm{m}$ displacement $(t=0.4 \mathrm{~s})$ to forelimbs and hindlimbs at stages TS22 and TS23. Stimuli displayed as external posterior and anterior views and through sections at presumptive bone regions (location marked with dotted horizontal line) displayed. Section orientations shown at top left, $a$ anterior, $p$ posterior, $d$ dorsal, $v$ ventral. Vertical scale bars (external views) $1 \mathrm{~mm}$
Patterns of biophysical stimuli predicted to result from muscle contractions were not found to be consistently higher in the presumptive bone regions of the humerus than in the femur over the three stages examined. At TS22 and TS23, peak levels of fluid flow, octahedral shear strain and combined stimulus in the presumptive bone regions were largely similar between the humerus and femur in animal \#1, as shown in Fig. 6, and were slightly higher (by between 20 and 50\%) in the humerus than in the femur in animal \#2 (data not shown). At TS24, results from both animals predict higher biophysical stimuli in the presumptive bone regions of the femur than in those of the humerus, as shown for animal \#1 in Fig. 6. These results indicate that muscle contractions in normal mouse embryos are unlikely to induce consistently higher biophysical stimuli in the humerus than in the femur. In simulations in which all muscles were activated simultaneously, there was again no trend to indicate that biophysical stimuli in the humerus were consistently higher than those in the femur (data not shown).

\subsection{Biophysical stimuli induced upon whole limb displacement}

Biophysical stimuli levels induced by a $10 \mu \mathrm{m}$ displacement at the distal end of the whole limb were consistently higher in the femur compared to the humerus, as shown in external views and sections taken at the presumptive bone regions at TS22 and TS23 in Fig. 7. Results shown are from
$0.4 \mathrm{~s}$ into the simulation, at the start of the hold phase of the applied displacement. Predicted peak octahedral shear strain levels in presumptive bone regions in the femur were roughly double those in the humerus, while fluid flows in the same region of the femur were also double, or greater than double, those in the humerus, as shown in Fig. 7. It is likely that the higher stimuli magnitudes predicted in the hindlimb are due to the angle and relative position of the rudiments within the limbs; note that the rudiments in the forelimb are contained mostly within one plane, while the hindlimb rudiments have a more complex geometry (Fig. 1). At the stages examined, the hindlimb is smaller than the forelimb (Table 1), which may also play a role in the higher stimuli levels in the femur as compared to the humerus when a displacement is applied. However, as the stimuli magnitudes in the whole limb models were similar between TS22 and TS23 (Fig. 7), despite the change in size (Table 1), we believe that shape, rather than size, is the primary source of the differences in stimuli between the rudiments. The magnitudes of the biophysical stimuli induced by the $10 \mu \mathrm{m}$ displacement were much higher than those induced by muscle contractions at equivalent stages, with peak octahedral shear strain values in the presumptive bone regions of up to $200 \mu$ strain and fluid velocities ranging from $9-16 \times 10^{-3} \mu \mathrm{m} / \mathrm{s}$ at TS23, compared with octahedral shear strains of $20 \mu$ strain and maximum fluid velocities of $1.5 \times 10^{-3} \mu \mathrm{m} / \mathrm{s}$ in the muscle contraction models at the same stage (Fig. 6). The peak levels of biophysical stimuli in the femur due to displacement 
roughly co-localised with the stimuli induced by muscle contractions, but this was not the case for the humerus (compare Fig. 6 with Fig. 7). Peak concentrations of stimuli were seen in the hip joint, and to a lesser extent in the knee and shoulder, while very low stimuli were predicted in the elbow joint (hip and shoulder shown in Fig. 7).

\subsubsection{Alternative model parameters}

Alternative displacement magnitudes of 1 and $50 \mu \mathrm{m}$ were examined. The same trends were observed with higher stimuli in the femur compared to the humerus in both cases. With a displacement of $1 \mu \mathrm{m}$, the magnitudes of the stimuli were closer to those induced by muscle contractions, with peak octahedral shear strains in the femoral presumptive bone region at TS22 of $20 \mu$ strain and a peak fluid velocity of $1.1 \times 10^{-3} \mu \mathrm{m} / \mathrm{s}$, compared to peak values due to muscle contractions at the same stage (data for $1 \mu \mathrm{m}$ not shown, maximum stimuli values due to muscle contractions as shown in Fig. 6). With a displacement of $50 \mu \mathrm{m}$, peak octahedral shear strains in the femoral presumptive bone region reached a maximum of $900 \mu$ strain, with peak fluid velocities in the same region of $70 \times 10^{-3} \mu \mathrm{m} / \mathrm{s}$ (data not shown). Simulations were also run with alternative directions of displacement (anterior to posterior and posterior to anterior) with a displacement of $10 \mu \mathrm{m}$, giving 8 anterior-posterior and 8 posterior-anterior simulations of each of the forelimb and hindlimb. In all simulations, the maximum fluid velocity was either similar or higher (by between 10 and $40 \%$ ) in the humerus than in the femur, apart from in the hip region, which always had very high fluid velocity (data not shown). In contrast, in 6 out of 8 simulations, there was higher (20\% on average) octahedral shear strain in the femur, while 7 out of 8 simulations also had a similar or higher $(25 \%$ on average) maximum combined stimulus value in the femur than in the humerus (data not shown). Therefore, even when the direction of the applied displacement is altered to one other than the most likely direction of passive movement, there is still a tendency towards higher maximum stimulus values in the femur compared to the humerus. When a load of $5 \mathrm{mN}$, instead of a displacement, was applied to the distal ends of the limbs, the maximum displacement of the forelimbs was 1.5-2.5 times greater than that of the hindlimbs (data not shown). In three out of the four models (two animals at TS22 and two at TS23), maximum octahedral shear strain values were slightly higher (by an average of 14\%) in the humerus than in the femur, while combined stimulus values were similar between the humerus and femur (data not shown). However, maximum fluid velocity values were still consistently slightly higher (by an average of 30\%) in the femur than in the humerus in all simulations (data not shown). Finally, whole limb analyses, in which cartilage material properties were used throughout, yielded similar results to simulations with decreased material properties for the joint, and the same trend of higher stimuli levels in the hindlimb than in the forelimb persisted (data not shown).

\section{Discussion}

In this study, we have investigated why some rudiments and joints are differentially affected when muscles are removed or reduced in genetically modified mice. We set out to test the hypothesis that mechanical influences are responsible for the differential effects seen in the developing limb and focussed our study on why the humerus is more severely affected than the femur when muscle is absent or reduced. Four out of five mechanosensitive genes with regulatory roles in ossification showed more extreme changes in the expression pattern in the humeri than in the femora of muscleless mutants; ColX, FGFr3, Ihh, and Runx2, while PTHrP expression appeared to be affected to the same degree in both the humeri and femora of mutant mice. Therefore, the differential effects of absent musculature were detectable at a molecular level. While the candidate genes examined may be indirectly influenced by mechanical stimuli, more extreme changes in the expression in the humerus than in the femur for four genes with mechanosensitive properties give a strong indication that the underlying mechanical environment may not be equal between these two rudiments in muscleless limb mice. We then used finite element analysis to predict and compare the biophysical stimuli active in the humerus and the femur at several stages of development. Finite element analyses of the humerus and femur predicted peaks of biophysical stimuli in the regions of prospective bone formation, as was predicted in our previous study of the chick tibiotarsus (Nowlan et al. 2008a), suggesting that a 'pulse' of biophysical stimuli contributes to the normal initiation of osteogenesis (Nowlan et al. 2008a). However, the analyses of the mouse humerus and femur showed that biophysical stimuli induced by muscle contractions were not consistently higher in the humerus than in the femur, and that, therefore, the removal of these stimuli alone could not explain the more severe effect on the humerus than in the femur. We then tested whether passive movements of the limbs due to forces external to the embryo could affect the rudiments to varying degrees using simplified morphologies of the whole limbs. The effects of movements of the mother or littermates (transferred to the mutant limb either via flow of the amniotic fluid or directly through pressure on the amnion) were modelled by applying a small displacement $(10 \mu \mathrm{m})$ towards the body at the distal end of the limb. The 'whole limb' analyses showed that higher biophysical stimuli are induced in the femur than in the humerus by a displacement to the distal end, and we propose that in the femur, the higher stimuli induced by external mechanical 
stimulation contribute to more normal skeletal development, despite the lack of muscle contractions and muscle contraction-induced movement. The whole limb analyses also predicted higher stimuli in the hip and knee joints compared to those in the shoulder and elbow, correlating with experimental evidence on the dependence on skeletal muscle of the elbow and shoulder but not of the knee (Nowlan et al. 2010a; Kahn et al. 2009). These results suggest that external forces, from movement of the mother or littermates, can explain why some rudiments and joints are affected more than others by the reduction or absence of skeletal muscle.

Some simplifications have been necessary for the computational analyses. For the 'whole limb' simulations, the shapes of the forelimbs and hindlimbs underwent simplification, particularly at the joint and zeugopod regions. However, as similar results were obtained in two different meshes from different animals at two timepoints, we are confident that the simplification of the morphologies does not affect our conclusions. We have examined the effects of a displacement of $10 \mu \mathrm{m}$ over $1.4 \mathrm{~s}$, but we cannot be sure of the duration, magnitude or direction of external mechanical stimulation on the embryonic limb. Therefore, we ran the models with alternative displacement values and directions and found that these analyses also yielded the same general trend of higher biophysical stimuli in the hindlimb compared to the forelimb regardless of the magnitude or direction of the displacement applied. Whole limb analyses in which a force, rather than a displacement, was applied to the limbs still resulted in higher levels of fluid velocity in the femur compared to those in the humerus. However, octahedral strain levels and combined stimulus levels were either similar or slightly higher in the humerus compared to the femur in three out of four cases. This result was most likely due to a much higher deformation of the forelimb than the hindlimb with the same applied force, and we propose that external forces are best modelled using displacement control rather than force control implementations. By TS23, cavitation has occurred in the major joints of the stylopod and zeugopod, but it is impossible to know how much (if any) articulation takes place in the joints at such an early stage of development, and we did not include any movement at the joints in the models. However, greater rotation is facilitated at the joints due to the reduced mechanical properties in those regions. While the mechanical properties of the mammalian interzone tissue are unavailable and were therefore estimated, alternative models in which cartilage tissue properties were used throughout the limb demonstrated that the Young's modulus of the joint regions did not affect the core finding of higher biophysical stimuli in the hindlimb than in the forelimb. It is likely that there is some relative movement at the joints at TS24, and this is why we did not model this stage.

Magnitudes of biophysical stimuli induced in the presumptive bone regions by passive movement were roughly ten times those induced by muscle contractile forces. However, it is important to consider that muscle contractions will cause movements of the limb, thereby inducing similar, or greater, biophysical stimuli than those predicted for a $10 \mu \mathrm{m}$ distal displacement of the limb. Therefore, muscle contractions in a normal mouse cause limb movements, and those movements induce biophysical stimuli in addition to the stimuli induced by the direct application of the muscle forces to the rudiments. The finite element results presented in this study would suggest that the biophysical stimuli induced by limb movement are more important for skeletal development than those induced by the direct application of muscle forces to the rudiments. However, in a muscleless mouse, the only source of limb movement is passive movement, and our results suggest that passive movements may be responsible for the differential effects absent limb musculature have on developing bones and joints (Nowlan et al. 2010a; Kahn et al. 2009). We have found that biophysical stimuli induced by passive movements are unequal between regions of the developing limbs, such as between the femur and humerus and between the knee and elbow. The angle and position of the limbs mean that higher biophysical stimuli are induced in the hindlimb than in the forelimb when a displacement is applied to the distal end of the limb. Our theory can also explain why some features in the muscleless limb mice have a variable degree of severity, for example, why only some muscleless mutants have incomplete calcification of the scapular blade (Nowlan et al. 2010a), as the amount and frequency of external displacements (and therefore the levels of stimuli induced) will vary depending on the activity levels of the mother, the size of the litter and perhaps even relative location in the uterus. The proposed theory can also explain why bone formation is significantly affected only in the scapula and humerus and not in other rudiments in reduced muscle mice (Nowlan et al. 2010a). In the reduced muscle mice, the additive combination of biophysical stimuli due to direct muscle forces, movement induced by muscle contraction and passive movements, is sufficient to give normal bone formation in the femur but not in the humerus, perhaps due to a higher contribution of biophysical stimuli from passive movements in the hindlimb than in the forelimb. While Kahn et al. (2009) have suggested that the range of effects seen in the joints of muscleless mice may be due to differences in the regulation of $\beta$-catenin, we suggest instead that varying levels of biophysical stimuli induced in the joints by external forces may contribute to the differential regulation of $\beta$-catenin, which has been shown to be involved in mechanoregulatory pathways (Lee et al. 2000).

In conclusion, we have investigated the role of biomechanical factors in the differential effects seen in mouse embryos with abnormal muscle development, where the humerus is more severely affected than the femur when limb muscle is reduced or absent. We have shown that mechanosensitive 
gene expression patterns are more severely affected in the humerus than in the femur of muscleless limb mice, indicating that the underlying mechanical environment may differ between the two rudiments despite the lack of skeletal muscle. Based on the predictions of our finite element analyses, we propose that passive movement induces a differential mechanical environment between regions of the developing skeleton when muscle is absent, thereby contributing to the differential effects seen in the developing bones and joints of muscleless and reduced muscle mutant mice. We propose that passive movements induced by external manipulation partially compensate for the effects of abnormal muscle development on skeletogenesis. This could have important implications for the treatment of in utero conditions where reduced movement affects bone and joint development, such as hemiplegic cerebral palsy (Roberts et al. 1994) and foetal akinesia deformation sequence (Hall 1986). Finally, our results suggest that biophysical stimuli induced by limb movements are the most important mechanical influence on the normal development of bones and joints, rather than the direct application of muscle forces to skeletal rudiments.

Acknowledgments We are grateful to Robert Hill, Edinburgh, UK, for RNA probes. This research was funded by the Wellcome Trust (083539/Z/07/Z), and by a grant from the French Embassy in Ireland (Service de Coopération et d'Action Culturelle). NCN was funded by a Marie Curie Intra European Fellowship within the 7th European Community Framework Programme and a fellowship from the Irish Research Council for Science, Engineering \& Technology. The Tajbakhsh laboratory was funded by the Institut Pasteur and AFM.

Open Access This article is distributed under the terms of the Creative Commons Attribution Noncommercial License which permits any noncommercial use, distribution, and reproduction in any medium, provided the original author(s) and source are credited.

\section{References}

Carter DR, Orr TE, Fyhrie DP, Schurman DJ (1987) Influences of mechanical stress on prenatal and postnatal skeletal development. Clin Orthop Relat Res 15(219):237-250

DeLaurier A, Burton N, Bennett M, Baldock R, Davidson D, Mohun T, Logan M (2008) The mouse limb anatomy atlas: an interactive 3D tool for studying embryonic limb patterning. BMC Dev Biol $8(1): 83$

Drachman DB, Sokóloff L (1966) The role of movement in embryonic joint formation. Dev Biol 14:401-420

Goldring MB, Tsuchimochi K, Ijiri K (2006) The control of chondrogenesis. J Cell Biochem 97(1): 33-44. doi:10.1002/jcb.20652

Gomez C, David V, Peet NM, Vico L, Chenu C, Malaval L, Skerry TM (2007) Absence of mechanical loading in utero influences bone mass and architecture but not innervation in Myod-Myf5-deficient mice. J Anat 210(3):259-271. doi:10.1111/j.1469-7580. 2007.00698.x

Hall BK, Herring SW (1990) Paralysis and growth of the musculoskeletal system in the embryonic chick. J Morphol 206(1):45-56
Hall JG (1986) Analysis of Pena Shokeir phenotype. Am J Med Genet 25(1):99-117

Hammond E, Donnenfeld AE (1995) Fetal Akinesia. Obstet Gynecol Surv 50(3):240-249

Heegaard JH, Beaupré GS, Carter DR (1999) Mechanically modulated cartilage growth may regulate joint surface morphogenesis. J Orthop Res 17(4):509-517

Henderson JH, Carter DR (2002) Mechanical induction in limb morphogenesis: the role of growth-generated strains and pressures. Bone 31(6):645-653

Hosseini A, Hogg DA (1991) The effects of paralysis on skeletal development in the chick embryo. I. General effects. J Anat 177:159168

Jackson RA, Kumarasuriyar A, Nurcombe V, Cool SM (2006) Longterm loading inhibits ERK1/2 phosphorylation and increases FGFR3 expression in MC3T3-E1 osteoblast cells. J Cell Physiol 209(3):894-904

Kahn J, Shwartz Y, Blitz E, Krief S, Sharir A, Breitel DA, Rattenbach R, Relaix F, Maire P, Rountree RB, Kingsley DM, Zelzer E (2009) Muscle contraction Is necessary to maintain joint progenitor cell fate. Dev Cell 16(5):734-743

Kassar-Duchossoy L, Gayraud-Morel B, Gomes D, Rocancourt D, Buckingham M, Shinin V, Tajbakhsh S (2004) Mrf4 determines skeletal muscle identity in Myf5: Myod double-mutant mice. Nature 431(7007):466-471. doi:10.1038/nature02876

Lacroix D, Prendergast PJ (2002) A mechano-regulation model for tissue differentiation during fracture healing: analysis of gap size and loading. J Biomech 35(9):1163-1171

Landmesser L, Morris DG (1975) The development of functional innervation in the hind limb of the chick embryo. J Physiol 249(2):301326

Lanske B, Karaplis AC, Lee K, Luz A, Vortkamp A, Pirro A, Karperien M, Defize LH, Ho C, Mulligan RC, Abou-Samra AB, Juppner H, Segre GV, Kronenberg HM (1996) PTH/PTHrP receptor in early development and Indian hedgehog-regulated bone growth. Science 273(5275):663-666

Lee HS, Millward-Sadler SJ, Wright MO, Nuki G, Salter DM (2000) Integrin and mechanosensitive ion channel-dependent tyrosine phosphorylation of focal adhesion proteins and beta-Catenin in human articular chondrocytes after mechanical stimulation. J Bone Min Res 15(8):1501-1509

Miller ME, Hangartner TN (1999) Temporary brittle bone disease: association with decreased fetal movement and osteopenia. Calcif Tissue Int 64(2): 137-143

Mitrovic D (1982) Development of the articular cavity in paralyzed chick embryos and in chick embryo limb buds cultured on chorioallantoic membranes. Acta Anat (Basel) 113(4):313-324

Murray PD, Drachman DB (1969) The role of movement in the development of joints and related structures: the head and neck in the chick embryo. J Embryol Exp Morphol 22(3):349-371

Ng AF, Yang YO, Wong RW, Hagg EU, Rabie AB (2006) Factors regulating condylar cartilage growth under repeated load application. Front Biosci 11:949-954

Nicole S, Diaz CC, Frugier T, Melki J (2002) Spinal muscular atrophy: recent advances and future prospects. Muscle Nerve 26(1):4-13

Nowlan NC, Murphy P, Prendergast PJ (2008a) A dynamic pattern of mechanical stimulation promotes ossification in avian embryonic long bones. J Biomech 41(2):249-258

Nowlan NC, Prendergast PJ, Murphy P (2008b) Identification of Mechanosensitive Genes during Embryonic Bone Formation. PLoS Comput Biol 4(12):e1000250. doi:10.1371/journal.pcbi.1000250

Nowlan NC, Bourdon C, Dumas G, Tajbakhsh S, Prendergast PJ, Murphy P (2010a) Developing bones are differentially affected by compromised skeletal muscle formation. Bone 46(5):1275-1285

Nowlan NC, Sharpe J, Roddy K, Prendergast PJ, Murphy P (2010b) Mechanobiology of embryonic skeletal development: insights 
from animal models. Birth Defects Res Part C Embryo Today Rev 90(3):203-213

Osborne AC, Lamb KJ, Lewthwaite JC, Dowthwaite GP, Pitsillides AA (2002) Short-term rigid and flaccid paralyses diminish growth of embryonic chick limbs and abrogate joint cavity formation but differentially preserve pre-cavitated joints. J Musculoskelet Neuronal Interact 2(5):448-456

Prendergast PJ, Huiskes R, Soballe K (1997) Biophysical stimuli on cells during tissue differentiation at implant interfaces. J Biomech 30(6):539-548

Provot S, Schipani E (2005) Molecular mechanisms of endochondral bone development. Biochem Biophys Res Commun 328(3):658665

Roberts CD, Vogtle L, Stevenson RD (1994) Effect Of Hemiplegia On Skeletal Maturation. J Pediat 125(5):824-828

Roddy KA, Kelly GM, van Es MH, Murphy P, Prendergast PJ (2011a) Dynamic patterns of mechanical stimulation co-localise with growth and cell proliferation during morphogenesis in the avian embryonic knee joint. J Biomech 44(1):143-149

Roddy KA, Prendergast PJ, Murphy P (2011b) Mechanical influences on morphogenesis of the knee joint revealed through morphological, molecular and computational analysis of immobilised embryos. PLoS one 6(2):e17526

Rodriguez JI, Garcia-Alix A, Palacios J, Paniagua R (1988a) Changes in the long bones due to fetal immobility caused by neuromuscular disease. A radiographic and histological study. J Bone Joint Surg Am 70(7):1052-1060

Rodriguez JI, Palacios J, Garcia-Alix A, Pastor I, Paniagua R (1988b) Effects of immobilization on fetal bone development. A morphometric study in newborns with congenital neuromuscular diseases with intrauterine onset. Calcif Tissue Int 43(6):335-339

Rot-Nikcevic I, Reddy T, Downing KJ, Belliveau AC, Hallgrimsson B, Hall BK, Kablar B (2006) Myf5 ${ }^{-/-}:$Myod $^{-/-}$amyogenic fetuses reveal the importance of early contraction and static loading by striated muscle in mouse skeletogenesis. Dev Genes Evol 216(1):1-9

Ruano-Gil D, Nardi-Vilardaga J, Tejedo-Mateu A (1978) Influence of extrinsic factors on the development of the articular system. Acta Anat (Basel) 101(1):36-44

Rudnicki MA, Schnegelsberg PN, Stead RH, Braun T, Arnold HH, Jaenisch R (1993) Myod or Myf-5 is required for the formation of skeletal muscle. Cell 75(7):1351-1359. doi:0092-8674(93)90621-V

Sarin VK, Carter DR (2000) Mechanobiology and joint conformity regulate endochondral ossification of sesamoids. J Orthop Res 18(5):706-712
Sharpe J, Ahlgren U, Perry P, Hill B, Ross A, Hecksher-Sorensen J, Baldock R, Davidson D (2002) Optical projection tomography as a tool for 3D microscopy and gene expression studies. Science 296(5567):541-545

Shefelbine SJ, Carter DR (2004) Mechanobiological predictions of growth front morphology in developmental hip dysplasia. J Orthop Res 22(2):346-352

Shiang R, Thompson L, Zhu Y, Church D, Fielder T, Bocian M, Winokur S, Wasmuth J (1994) Mutations in the transmembrane domain of FGFR3 cause the most common genetic form of dwarfism, achondroplasia. Cell 78(2):335-342

Stevens SS, Beaupré GS, Carter DR (1999) Computer model of endochondral growth and ossification in long bones: biological and mechanobiological influences. J Orthop Res 17(5):646-653

Sundaramurthy S, Mao JJ (2006) Modulation of endochondral development of the distal femoral condyle by mechanical loading. J Orthop Res 24(2):229-241

Suzue T (1996) Movements of mouse fetuses in early stages of neural development studied in vitro. Neurosci Lett 218(2):131-134

Tajbakhsh S, Bober E, Babinet C, Pournin S, Arnold H, Buckingham M (1996) Gene targeting the myf-5 locus with nlacZ reveals expression of this myogenic factor in mature skeletal muscle fibres as well as early embryonic muscle. Dev Dyn 206(3):291-300

Tajbakhsh S, Rocancourt D, Cossu G, Buckingham M (1997) Redefining the genetic hierarchies controlling skeletal myogenesis: Pax-3 and Myf-5 act upstream of Myod. Cell 89(1):127-138

Tanaka N, Ohno S, Honda K, Tanimoto K, Doi T, Ohno-Nakahara M, Tafolla E, Kapila S, Tanne K (2005) Cyclic mechanical strain regulates the PTHrP expression in cultured chondrocytes via activation of the Ca2+ channel. J Dent Res 84(1):64-68

Tanck E, Blankevoort L, Haaijman A, Burger EH, Huiskes R (2000) Influence of muscular activity on local mineralization patterns in metatarsals of the embryonic mouse. J Orthop Res 18(4):613-619

Tanck E, Van Donkelaar CC, Jepsen KJ, Goldstein SA, Weinans H, Burger EH, Huiskes R (2004) The mechanical consequences of mineralization in embryonic bone. Bone 35(1):186-190

Theiler K (1989) The house mouse: atlas of embryonic development. 2nd edn. Springer, New York

Wesstrom G, Bensch J, Schollin J (1986) Congenital myotonic dystrophy. Acta Paediat 75(5):849-854

Yoshida C, Furuichi T, Fujita T, Fukuyama R, Kanatani N, Kobayashi S, Satake M, Takada K, Komori T (2002) Core-binding factor interacts with Runx 2 and is required for skeletal development. Nature Genet 32(4):633-638 